Jean-Pol Tassin

François Trovero

Paul Vezina

Gérard Blanc

Jacques Glowinski Denis Hervé

\section{ADRESSE}

J.P. Tassin: directeur de recherche à l'Inserm. F. Trovero: docteur ès sciences, université ParisVI. P. Vezina: professeur, université de Chicago (Etats-Unis). G. Blanc: ingénieur au CNRS.

J. Glowinski: professeur au Collège de France. D. Hervé : chargé de recherche à l'inserm. Chaire de neuropharmacologie, Inserm U. 114 Collège de France, 11, place Marcelin-Berthelot, 75231 Paris Cedex 05, France.

\title{
L'hétéro-régulation des récepteurs ou la présence d'une relation fonctionnelle entre deux ensembles neuronaux
}

Les neurones du système nerveux central portent plusieurs types de récepteurs, chacun de ces récepteurs étant sensible à l'un des nombreux transmetteurs libérés par les différentes fibres afférentes. La liaison d'un neuromédiateur à son récepteur homologue peut donc modifier, par l'intermédiaire de mécanismes intracellulaires, la sensibilité ou la densité des autres récepteurs, hétérologues, portés par la même cellule. C'est ainsi, par exemple, que la transmission et la sensibilité des récepteurs dopaminergiques corticaux et sous-corticaux de type D1 est modulée par des afférences libérant d'autres neurotransmetteurs, tels que l'acide glutamique ou la noradrénaline. Nous proposons que la mise en évidence in vivo de ces interactions hétérologues implique l'existence d'une relation fonctionnelle privilégiée entre les ensembles neuronaux mis en jeu.

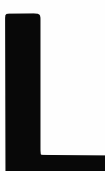

a complexité anatomique du système nerveux central est telle qu'il paraît peu probable qu'une analyse même très élaborée de tous les contacts synaptiques suffise à élucider les relations fonctionnelles entre les différents ensembles neuronaux. Il semble, en revanche, que l'analyse des facteurs intervenant in vivo dans la régulation des récepteurs centraux puisse apporter des informations sur ces interactions.

A la périphérie, il est bien connu que la dégénérescence des fibres afférentes cholinergiques de la jonction neuro-musculaire entraîne une hypersensibilité de dénervation des récepteurs post-synaptiques de l'acétylcholine. Dans le système nerveux central, le développement d'une hypersensibilité de récepteurs après dénervation répond à des règles plus complexes. En effet, la destruction d'un groupe d'afférences, et de façon plus générale l'interruption de la transmission d'un neuromédiateur, n'entraîne pas nécessairement l'apparition d'une hypersensibilité des récepteurs correspondants. De fait, chaque cellule reçoit un grand nombre de fibres nerveuses et présente sur sa membrane plusieurs types de récepteurs. La liaison d'un neuromédiateur à son récepteur (homologue) peut alors modifier la 


\section{RÉFÉRENCES}

1. Hervé $D$, Trovero F, Blanc G, Vézina $P$, Glowinski J, Tassin JP. Involvement of dopamine neurons in the regulation of $\beta$-adrenergic receptors sensitivity in rat prefrontal cortex. Neuroscience 1990; 54 : 1864-9.

2. Hervé D, Tassin JP, Studler JM, Dana C, Kitabgi P, Vincent JP, et al. Dopaminergic control of $125 \mathrm{I}$-labeled neurotensin binding
site density in corticolimbic structures of the rat brain. Proc Natl Acad Sci USA 1986; 83: 6203-7.

3. Trovero F, Hervé D, Desban M, Glowinski J, Tassin JP. Striatal opiate $\mu$-receptors are not located on dopamine nerve endings in the rat. Neuroscience $1990 ; 2$ : 313-21.

4. Fuxe K, Agnati LF, eds. Receptor-receptor interactions: a new intramembrane integrative mechanism. Wenner-Gren International Symposium Series, vol. 48. London : MacMillan Press LTD 1987.

5. Björklund A, Lindvall $O$. Dopamine containing systems in the CNS. In: Björklund A, Hökfelt T, eds. Handbook of chemical neuroanatomy. Amsterdam : Elsevier, 1984: 55-122.

6. Akert K. Comparative anatomy of the frontal cortex and thalamocortical connections. In : Warren JM, Akert $\mathrm{K}$, eds. The frontal and agranular cortex and behaviour. New York: McGraw-Hill, 1964: 372-96.

7. Divac I, Björklund A, Lindvall O, Passingham RE. Converging projections from the medio-dorsal nucleus and mesencephalic dopaminergic neurons to the neocortex in three species. J Compar Neurol 1978: 59-72.

8. Lavielle S, Tassin IP, Thierry AM, Blanc G, Hervé D, Barthélémy C, et al. Blockade by benzodiazepines of the selective high increase in DA turnover induced by stress in mesocortical DA neurons in the rat. Brain Res $1979 ; 168$ : 585-94.

9. Blanc G, Hervé D, Simon H, Lisoprawski A, Glowinski J, Tassin JP. Response to stres of mesocortico-prefrontal DA neurons in rats after long-term isolation. Nature 1980 $284: 265-7$.

10. Le Moal M, Cardo B, Stinus L. Influence of ventral mesencephalic lesions on various spontaneous and conditional behaviours in the rat. Physiol Behav 1969; 4: 567-74.

11. Pycock CJ, Kerwin RW, Carter CJ. Effect of 6-hydroxydopamine lesions of the medial prefrontal cortex on neurotransmitter systems in subcortical sites in the rat. $J$ Neuro chem $1980 ; 34: 91-9$.

12. Louilot A, Simon H, Taghzouti K, Le Moal M. Modulation of dopaminergic activity in the nucleus accumbens following facilitation or blockade of the dopaminergic transmission in the amygdala: a study by in vivo differential pulse voltametry. Brain Res vivo differential densité ou la sensibilité d'un ou plusieurs autres récepteurs (hétérologues) situés sur la même cellule. Par conséquent, dans le système nerveux central, l'absence d'une hypersensibilité de dénervation, lors de l'interruption d'une neurotransmission, peut signifier la présence d'une compensation due à la modification d'activité d'autres neurones, hétérologues. De même, si une hypersensibilité de dénervation n'apparaît plus lorsqu'une autre neurotransmission a été interrompue simultanément, cela peut indiquer une relation fonctionnelle antagoniste entre les deux ensembles neuronaux.

Notre hypothèse propose donc que la démonstration in vivo d'une influence hétérologue à long terme dans la régulation d'un récepteur implique l'existence d'une relation fonctionnelle privilégiée entre les deux ensembles neuronaux mis en jeu. Nous avons choisi de présenter dans cette revue nos résultats sur l'hétéro-régulation d'un sous-type de récepteur dopaminergique, le récepteur D1, couplé positivement à l'adénylyl cyclase. Il se trouve, en effet, que la régulation de ce récepteur a été étudiée dans différentes structures, corticales et sous-corticales, et que, dans plusieurs cas, les modifications d'activité de l'adénylyl cyclase couplée positivement à la dopamine se sont avérées correspondre à des expressions comportementales. Ce phénomène d'hétéro-régulation n'est cependant pas spécifique $d u$ récepteur D1. Il a été retrouvé, par exemple, pour l'activité de l'adénylyl cyclase couplée au récepteur $\beta$-adrénergique [1], mais aussi à l'occasion de l'étude de la régulation de la densité des récepteurs $\mu$-opiacés ou neurotensinergiques par des fibres dopaminergiques $[2,3]$. D'autres interactions entre récepteurs hétérologues, correspondant le plus souvent à des phénomènes directs ou intervenant à très court terme, ont été rassemblés dans un ouvrage [4].

\section{Interrelation fonctionnelle des voies dopaminergiques ascendantes}

Chez le rat, l'aire tegmentale ventrale et la substance noire, deux structures mésencéphaliques, contiennent les corps cellulaires de l'essentiel des neurones dopaminergiques ascendants [5] (figure 1)

Dans cet ensemble aire tegmentale ventrale-substance noire, $3 \%$ seulement des neurones dopaminergiques se projettent sur le cortex préfrontal, cingulaire et entorhinal. Malgré leur petit nombre, ces neurones mésocorticaux présentent plusieurs caractéristiques qui suggèrent qu'ils jouent un rôle essentiel dans la régulation de l'émotivité. Ces neurones dopaminergiques innervent une zone corticale restreinte, elle-même en relation avec le noyau médio-dorsal du thalamus, une connexion qui définit les aires corticales responsables des processus cognitifs et émotifs $[6,7]$. En outre, des situations anxiogènes augmentent l'utilisation de la dopamine dans ces aires corticales et cet effet est bloqué par l'administration préalable de benzodiazépines [8]. Par ailleurs, quelques semaines d'isolement suffisent à réduire l'activité des neurones dopaminergiques méso-corticaux et les rendent hypersensibles à des stimulations anxiogènes [9]. Enfin, la destruction de ces neurones dopaminergiques semble responsable du développement d'un syndrome caractérisé par une hyperactivité locomotrice, la disparition de l'alternance spontanée et, d'une façon générale, la perte des fonctions inhibitrices comportementales autorisant la réalisation de tâches coordonnées [10].

Les neurones dopaminergiques méso-corticaux ne doivent cependant pas être considérés comme une entité isolée. En effet, plusieurs résultats indiquent que les différentes cellules dopaminergiques qui innervent les structures corticales et sous-corticales sont en interrelation. Ainsi, en 1980, Pycock et al. ont montré que la destruction des fibres dopaminergiques du cortex préfrontal provoquait une augmentation de l'utilisation de la dopamine dans le noyau accumbens [11]. Même si certaines de ces données n'ont pu être reproduites dans d'autres laboratoires, les relations qu'elles suggéraient entre les neurones dopaminergiques ont été vérifiées dans différents modèles expérimentaux. Par exemple, l'interruption de la transmission dopaminergique dans l'amygdale entraîne une aug- 
mentation de l'utilisation de la clopamine dans le noyau accumbens et une diminution dans le cortex préfrontal $[12,13]$. De même, une confirmation indirecte de ces interrelations a été obtenue en mesurant l'utilisation de la dopamine dans des structures corticales et sous-corticales à la suite de lésions de différents noyaux (raphés dorsal et médian, habenula, locus coeruleus) qui se projettent dans le complexe aire tegmentale ventralesubstance noire [14-17]. Ces dernières années, nous nous sommes particulièrement attachés à l'étude de l'influence de l'activité des cellules dopaminergiques méso-corticales sur les transmissions dopaminergiques sous-corticales.

\section{Influence corticale dopaminergique sur les fonctions dopaminergiques du noyau accumbens et du striatum}

Contrôle de la transmission dopaminergique dans le noyau accumbens

Il est généralement admis que l'hyperactivité locomotrice induite par une injection d'amphétamine dans le noyau accumbens est due à une augmentation de la libération de dopamine dans ce noyau [18]. Par ailleurs, des données indirectes, comme l'effet d'une lésion électrolytique de l'aire tegmentale ventrale, suggèrent qu'une baisse de la transmission dopaminergique corticale augmente l'activité locomotrice [19]. Nous avons donc cherché à démontrer directement cette influence corticale en mesurant l'activité locomotrice chez des rats soumis à des injections simultanées d'amphétamine dans le cortex préfrontal et le noyau accumbens. Comme attendu, une injection bilatérale d'amphétamine dans le noyau accumbens augmente l'activité locomotrice des animaux. En revanche, si l'amphétamine est in jectée simultanément dans le cortex préfrontal et le noyau accumbens, l'hyperactivité locomotrice est réduite de $42 \%$ [20]. Nous avons aussi montré que cet effet passait par une stimulation des récepteurs dopaminergiques corticaux de type Dl puisque l'injection bilatérale d'un antagoniste des récepteurs $\mathrm{Dl}$, le $m / s n^{\circ} 6$, vol. 11 , juin 95
Figure 1. Schéma des voies anatomiques en relation avec l'hétérorégulation des récepteurs $D 1$. Les neurones dopaminergiques (DA) ascendants dont les corps cellulaires se situent dans le mésencéphale (aire tegmentale ventrale et substance noire) innervent de façon divergente le cortex préfrontal et les structures sous-corticales (noyau accumbens [N. Acc.] et striatum). Les neurones dopaminergiques mésocorticaux modifient l'activité des neurones glutamatergiques du cortex préfrontal qui projettent dans le noyau accumbens et le striatum. Ces contacts peuvent être directs ou indirects par l'intermédiaire d'interneurones GABAergiques. Les récepteurs D1 des structures sous-corticales sont sous le double contrôle des fibres dopaminergiques issues $d u$ mésencéphale (homologues) et des efférences glutamatergiques corticales (hétérologues). Enfin, les neurones noradrénergiques issus du locus coeruleus, en stimulant les récepteurs $\alpha 1$-adrénergiques corticaux, inhibent la transmission dopaminergique corticale en agissant sur les récepteurs D1. VTA: aire tegmentale ventrale; SN: substance noire.

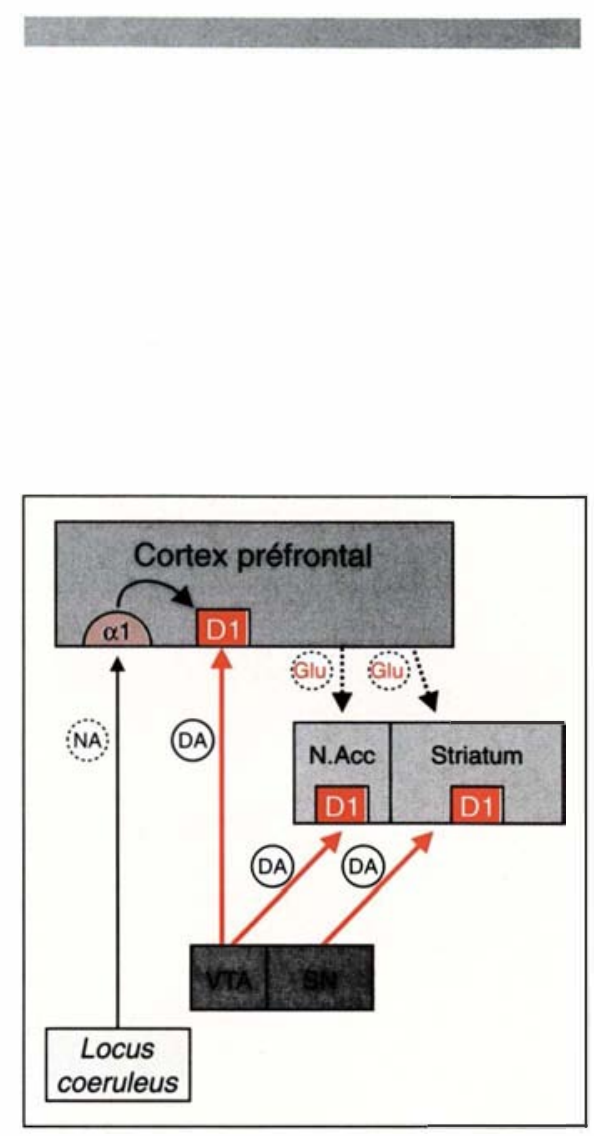

SCH 23390, augmente de $110 \%$ l'activité locomotrice induite par l'injection d'amphétamine dans le noyau accumbens. L'injection corticale d'un antagoniste des récepteurs dopaminergiques de type D2, le sulpiride, est sans effet. Ces expériences apportent ainsi une preuve directe du rôle inhibiteur de la stimulation des récepteurs Dl corticaux sur l'hyperactivité locomotrice induite par une stimulation des récepteurs dopaminergiques du noyau accumbens.

Contrôle de la transmission dopaminergique dans le striatum

Il est bien connu que la destruction unilatérale de l'innervation dopaminergique d'un striatum entraîne, quatre semaines après la lésion, un comportement de rotation lorsque les animaux sont traités par des agonistes dopaminergiques [21]. Cet effet est considéré comme étant dû au développement d'une hypersensibilité des récepteurs dopaminergiques dans le striatum lésé, le traitement par un agoniste dopaminergique permettant de révéler le déséquilibre induit par la lésion. Si une destruction bilatérale de l'innervation dopaminergique corticale est surajoutée à la destruction unilatérale de l'innervation dopaminergique du striatum, le comportement de rotation induit par un agoniste des récepteurs dopaminergiques de type D1, le SKF 38393, diminue de $56 \%$. Dans ces deux exemples, une augmentation ou une diminution de la transmission dopaminergique corticale s'oppose aux effets obtenus par une augmentation de la transmission dopaminergique sous-corticale. Dans 


\section{RÉFÉRENCES}

13. Simon H, Taghzouti K, Gozlan H, Studler JM, Louilot A, Hervé $\mathrm{D}$, et al. Lesion of dopaminergic terminals in the amygdala produces enhanced locomotor response to D-amphetamine and opposite changes in dopaminergic activity in prefrontal cortex and nucleus accumbens. Brain Res 1988; 447 . 335-40.

14. Hervé D, Simon H, Blanc G, Lisoprawski A, Le Moal M, Glowinski I, et al. Increased utilization of dopamine in the nucleus accumbens but not in the cerebral cortex after dorsal raphe lesion in the rat. Neurosci Lett $1979 ; 15$ : $877-88$

15. Hervé D, Simon H, Blanc G, Glowinski J, Tassin JP. Opposite changes in dopamine utilization in the nucleus accumbens and the frontal cortex after electrolytic lesion of the median raphe in the rat. Brain Res 1981 ; 216: 422-8.

16. Hervé D, Blanc G, Glowinski J, Tassin JP. Reduction of dopamine utilization in the prefrontal cortex but not in the nucleus accumbens after selective destruction of noradrenergic fibers innervating the ventral tegmental area in the rat. Brain Res $1982 ; 237$ : 510-6.

17. Lisoprawski A, Hervé D, Blanc G, Glowinski J, Tassin JP. Selective activation of the mesocortico-frontal dopaminergic neurons induced by the lesion of the habenula in the rat. Brain Res $1980 ; 183: 229-34$.

18. Kelly PH, Seviour PW, Iversen SD. Amphetamine and apomorphine responses in the rat following 6-OHDA lesions of the nucleus accumbens septi and corpus striatum. Brain Res 1975; 94 : 507-22.

19. Tassin JP, Stinus L, Simon H, Blanc G, Thierry AM, Lavielle $S$, et al. Relationship between the locomotor hyperactivity induced by Al0 lesions and the destruction of fronto-cortical DA innervation in the rat. Brain Res 1978; 141 : $267-81$.

20. Vézina P, Blanc G, Glowinski, Tassin JP. Opposed behavioural outputs of increased dopamine transmission in prefrontocortical and subcortical areas: a role for the cortical D1 dopamine receptor. Eur J Neurosci 1991 ; 3: 1001-7.

21. Ungerstedt U, Arbuthnott G. Quantitative recording of rotational behaviour in rats after 6-hydroxydopamine of the nigro-striatal dopamine system. Brain Res 1970; 24 : 485-93.

22. Tassin JP, Simon H, Hervé D, Blanc G, Le Moal M, Glowinski J, et al. Non-dopaminergic fibres may regulate dopamine-sensitive adenylate cyclase in the prefrontal cortex and the nucleus accumbens. Nature 1982 ;

le premier cas, une stimulation de la transmission dopaminergique corticale inhibe le comportement induit par une augmentation de la transmission sous-corticale. Dans le second, la destruction de l'innervation dopaminergique corticale diminue ou compense l'effet dû à la destruction de l'innervation dopaminergique souscorticale. Dans les deux cas, la transmission dopaminergique corticale agit de manière opposée à celle des transmissions dopaminergiques souscorticales. Ces relations fonctionnelles ont pu être retrouvées en étudiant la régulation des récepteurs dopaminergiques de type Dl dans le noyau accumbens et le striatum.

\section{Hétéro-régulation des récepteurs D1 dans le noyau accumbens}

L'étude de la régulation des récepteurs D1 dans le noyau accumbens a été réalisée en détruisant les neurones dopaminergiques ascendants par une lésion bilatérale électrolytique ou chimique (à la 6-hydroxydopamine) de l'aire tegmentale ventrale. Six semaines après l'un ou l'autre type de lésion, la mesure de l'activité de l'adénylyl cyclase stimulée par la dopamine dans des homogénats du noyau accumbens permettait de déterminer les modifications de couplage des récepteurs Dl. Bien que les taux de dopamine aient été diminués de plus de $90 \%$ après ces lésions, nous n'avons pas retrouvé de modification de l'activité adénylyl cyclase sensible à la dopamine dans les noyaux accumbens dénervés [22, 23]. Compte tenu des résultats comportementaux que nous venons de mentionner, nous avons fait l'hypothèse que cette absence d'hypersensibilité de dénervation était due à une influence corticale. En effet, les deux types de lésion de l'aire tegmentale ventrale, électrolytique ou chimique, avaient détruit les fibres dopaminergiques corticales et vraisemblablement modifié l'activité des neurones glutamatergiques cortico-sous-corticaux (figure 1). Nous avons pu vérifier cette hypothèse en préparant des animaux qui avaient subi à la fois une lésion chimique de l'aire tegmentale ventrale et une ablation bilatérale du cortex préfrontal. Chez les animaux porteurs de ces doubles lésions, l'activité de l'adénylyl cyclase stimulée par la dopamine dans les noyaux accumbens était alors augmentée de 52\% [23]. Cette expérience indique que les neurones glutamatergiques efférents du cortex préfrontal peuvent, lorsqu'ils ne sont plus sous le contrôle des neurones dopaminergiques, éviter le développement de l'hypersensibilité de dénervation des récepteurs Dl du noyau accumbens. Cette absence d'hypersensibilité peut être interprétée comme le résultat d'une compensation fonctionnelle du déficit dopaminergique induit lors de la dénervation du noyau accumbens. L'activité des neurones glutamatergiques s'est modifiée de telle façon que l'hypersensibilité des récepteurs D1 n'est plus nécessaire.

Nous proposons en effet que, quelle que soit son origine, l'hypersensibilité des récepteurs D1 dans une structure signifie que les cellules qui portent les récepteurs Dl sont devenues incapables de traiter correctement l'information dopaminergique qu'elles pourraient recevoir. L'absence de l'hypersensibilité de dénervation signifierait alors que le déficit fonctionnel induit par la dénervation a été compensé par la modification d'activité d'autres cellules, hétérologues.

L'expérience que nous venons de décrire indiquerait donc que les projections dopaminergiques du cortex et du noyau accumbens jouent des rôles opposés sur les efférences comportementales du noyau accumbens sur lesquelles elles interviennent. Des résultats semblables ont été obtenus sur l'hétéro-régulation des récepteurs Dl du striatum.

\section{Hétéro-régulation des récepteurs $D 1$ du striatum}

La dénervation bilatérale par la 6-hydroxydopamine des fibres dopaminergiques du striatum ne permettant pas de maintenir les animaux en bonne condition, nous avons effectué une destruction unilatérale des neurones dopaminergiques de la substance noire. Six semaines après la lésion, l'activité adénylyl cyclase sensible à la dopamine était augmentée de $45 \%$ par rapport aux témoins dans le striatum antéro-médian [24]. Afin d'obtenir une dénervation aussi 
complète que possible du striatum, l'injection de la neurotoxine avait été réalisée légèrement antérieurement à la substance noire, dans la zone où toutes les fibres dopaminergiques se rassemblent. Les neurones dopaminergiques innervant le cortex avaient donc été détruits simultanément. Néanmoins, puisque la lésion des fibres dopaminergiques ascendantes n'avait été effectuée qu'unilatéralement, seule la partie du cortex ipsilatérale à la lésion était déplétée en dopamine. Par ailleurs, des études anatomiques nous avaient permis de constater que la partie antéro-médiane du striatum recevait des afférences issues de neurones glutamatergiques également répartis dans les cortex préfrontal ipsi- et controlatéral à la lésion. Cela signifiait donc que, chez les rats lésés, la moitié des neurones glutamatergiques du cortex qui innervent la partie antéro-médiane du striatum restait sous le contrôle de fibres dopaminergiques corticales. Lorsque ces fibres dopaminergiques corticales controlatérales ont été détruites par une injection de 6hydroxydopamine, la lésion unilatérale des fibres dopaminergiques du striatum n'a plus entraîné d'hypersensibilité de dénervation des récepteurs Dl du striatum antéro-médian [24].

Ces deux expériences, réalisées sur le noyau accumbens et le striatum, montrent bien les relations fonctionnelles qui lient les transmissions dopaminergiques corticales et sous-corticales. En effet, elles indiquent que les conséquences de la dénervation dopaminergique d'une structure sous-corticale n'apparaissent sous la forme d'une hypersensibilité de dénervation que si les fibres dopaminergiques corticales sont maintenues intactes ou si la voie cortico-sous-corticale est détruite. En d'autres termes, la modification de l'activité des fibres dopaminergiques corticales ou leur destruction permet de compenser le déficit induit par une dénervation dopaminergique souscorticale.

D'autres résultats nous ont permis de préciser les relations fonctionnelles qui existent entre ces structures corticales et sous-corticales. En effet, les récepteurs Dl du cortex préfrontal, et par conséquent la transmission dopaminergique corticale, sont eux $\mathrm{m} / \mathrm{s} n^{\circ} 6$, vol. 11, juin 95 aussi soumis au contrôle de fibres hétérologues.

\section{Rôle fonctionnel et hétéro-régulation des récepteurs $D 1$ corticaux}

\section{Importance de la transmission nora- drénergique corticale}

Comme nous l'avons déjà évoqué, la destruction de l'innervation dopaminergique corticale par électrocoagulation des corps cellulaires dopaminergiques de l'aire tegmentale ventrale entraîne un syndrome comportemental qui se manifeste principalement chez le rat par le développement d'une hyperactivité locomotrice et la disparition du comportement d'alternance spontanée [10]. Des études de corrélation ont par ailleurs montré que le syndrome est d'autant plus important que les concentrations de dopamine corticale sont réduites et que les récepteurs D1 du cortex sont hypersensibles [19, 22]. Curieusement, la destruction chimique des fibres dopaminergiques par une injection de 6-hydroxydopamine au niveau de l'aire tegmentale ventrale n'entraîne pas de tels symptômes, en dépit d'une baisse importante de dopamine au niveau cortical. De façon intéressante, il n'est pas non plus possible de détecter, chez ces animaux lésés, d'hypersensibilité des récepteurs Dl corticaux.

Outre la lésion des fibres dopaminergiques, la neurotoxine injectée dans l'aire tegmentale ventrale détruit également par diffusion les fibres noradrénergiques ascendantes. La présence d'une innervation noradrénergique semble donc nécessaire à l'apparition du syndrome de l'aire tegmentale ventrale. De fait, des expériences complémentaires indiquent que la destruction sélective de l'innervation noradrénergique abolit l'apparition du syndrome induit par la lésion électrolytique de l'aire tegmentale ventrale [25]. Ces expériences de lésion ont donc montré, dans un premier temps, le rôle permissif de l'innervation noradrénergique dans l'expression des déficits comportementaux induits par une dénervation dopaminergique corticale.

Dans un second temps, l'ensemble de ces expériences de dénervation a permis de mettre en évidence les mécanismes de régulation hétérologue des récepteurs D1 corticaux. Nous avons, en effet, vérifié que, comme dans le cas du syndrome de l'aire tegmentale ventrale, c'est l'absence des fibres noradrénergiques qui bloque le développement de l'hypersensibilité de dénervation des récepteurs Dl corticaux [26]. Des études biochimiques et comportementales complémentaires ont permis de caractériser le sous-type de récepteur noradrénergique dont la stimulation semble intervenir sur la régulation du récepteur D1 cortical. L'hyperactivité locomotrice induite par la lésion électrolytique de l'aire tegmentale ventrale peut, en effet, être totalement abolie par l'injection périphérique de prazosin, un antagoniste sélectif des récepteurs noradrénergiques de type $\alpha 1$ [27]. La dose de prazosin utilisée $(0,5 \mathrm{mg} / \mathrm{kg}$ par voie intrapéritonéale) n'affecte pas l'activité locomotrice des rats témoins. Ce résultat indique que le rôle permissif de la transmission noradrénergique dans le développement et le maintien des déficits comportementaux du syndrome de l'aire tegmentale ventrale sont relayés par la stimulation des récepteurs $\alpha 1$-adrénergiques. Des expériences complémentaires montrent que l'interaction entre les transmissions dopaminergiques et noradrénergiques aboutissant à l'expression de ce syndrome est localisée au niveau du cortex préfrontal. En effet, une injection de prazosin dans le cortex préfrontal à l'aide de canules placées à demeure sur le crâne de l'animal peut abolir une hyperactivité locomotrice induite par une injection d'amphétamine dans le noyau accumbens [28]. Étant donné le rôle inhibiteur de la transmission dopaminergique corticale de type Dl sur le comportement locomoteur [20], il est vraisemblable que le blocage des récepteurs $\alpha 1$-adrénergiques par le prazosin aboutisse à une facilitation de la transmission dopaminergique corticale. Cette facilitation entraînerait une modification de l'activité des fibres glutamatergiques excitatrices qui, en se projetant dans les structures sous-corticales, seraient responsables des modifications de sensibilité des récepteurs Dl de ces structures. 
Étude biochimique de l'interaction des récepteurs $\alpha 1$-adrénergiques et Dl dans le cortex préfrontal

\section{RÉFÉRENCES}

23. Reibaud M, Blanc G, Studler JM, Glowinski J, Tassin JP. Non-dopaminergic prefronto-cortical efferents modulate DI receptors in the nucleus accumbens. Brain Res $1984 ; 305: 43-50$

24. Hervé D, Trovero F, Blanc G, Thierry AM, Glowinski J, Tassin JP. Non-dopaminergic prefronto-cortical efferent fibers modulate $\mathrm{D} 1$ receptor denervation supersensitivity in specific regions of the rat striatum. I Neurosci $1989 ; 9$ : 3699-708.

25. Taghzouti K, Simon H, Hervé D, Blanc G, Studler JM, Glowinski J, et al. Behavioural deficits induced by an electrolytic lesion of the rat ventral mesencephalic tegmentum are corrected by a superimposed lesion of the dorsal noradrenergic system. Brain Res $1988 ; 440: 172-6$

26. Tassin JP, Studler JM, Hervé D, Blanc G, Le Moal M, Glowinski J. Contribution of noradrenergic neurons to the regulation of dopaminergic (D1) receptor denervation supersensitivity in rat prefrontal cortex. $J$ Neurochem 1986; 46 : 243-8.

27. Trovero F, Blanc G, Hervé D, Vézina $P$, Glowinski J, Tassin JP. Contribution of an $\alpha$ l-adrenergic receptor subtype to the expression of the "ventral tegmental area syndrome". Neuroscience 1992; 47: 69-76.

28. Blanc $G$, Trovero $F$, Vézina $P$, Hervé $D$ Godeheu AM, Glowinski J, et al. Blockade of prefronto-cortical $\alpha 1$-adrenergic receptors prevents locomotor hyperactivity induced by subcortical amphetamine injection. Eur J Neurosci $1994 ; 6$ : 293-8.

29. Trovero F, Hervé D, Blanc G, Glowinski J. Tassin JP. In vivo partial inactivation of dopamine D1 receptors induces hypersensitivity of cortical dopamine-sensitive adenylate cyclase: permissive role of $\alpha 1$-adrenergic receptors. Neurochem $1992 ; 59: 331-7$.

30. Trovero F, Marin P, Tassin JP, Premont J, Glowinski J. Accelerated resensitization of the Dl dopamine receptor-mediated response in cultured cortical and striatal neurons from the rat: respective role of $\alpha 1$ adrenergic and $\mathrm{N}$-methyl aspartate receptors. J Neurosci $1994 ; 14: 6208-8$.

31. Freund TF, Powell JF, Smith AD. Tyrosine hydroxylase-immunoreactive boutons in synaptic contact with identified striatonigral neurons with particular reference to dendritic spines. Neuroscience 1984; 13 : $1189-215$

32. Brown JR, Arbuthnott GW. The electrophysiology of dopamine (D2) receptors: a study of the action of DA on cortico-striatal transmission. Neuroscience $1983 ; 10: 349-55$.

33. Taghzouti K, Le Moal M, Simon H. Suppression of noradrenergic innervation compensates for behavioural deficits induced by lesion of dopaminergic terminals in the
Une première étude a été réalisée en utilisant un agent bloquant irréversiblement les récepteurs Dl, l'EEDQ (N-ethoxycarbonyl-2-ethoxy-1,2-dihydroquinoline). Injecté chez l'animal à très faibles doses, cet agent bloque une petite partie $(25 \%)$ des récepteurs Dl corticaux, provoquant ainsi une hypersensibilité des récepteurs résiduels en augmentant leur couplage à l'adénylyl cyclase. Cette hyperclique (AMPc). sensibilité n'apparaît pas chez des animaux préalablement traités avec l'antagoniste sélectif des récepteurs $\alpha$ l-adrénergiques, le prazosin [29]. Ce résultat obtenu in vivo montre que le blocage sélectif de la transmission noradrénergique corticale au niveau des récepteurs $\alpha 1$-adrénergiques empêche le développement d'une hypersensibilité des récepteurs Dl

Très récemment, en utilisant des neurones de cortex embryonnaires en culture, nous avons pu montrer que la stimulation des récepteurs $\alpha 1$ -

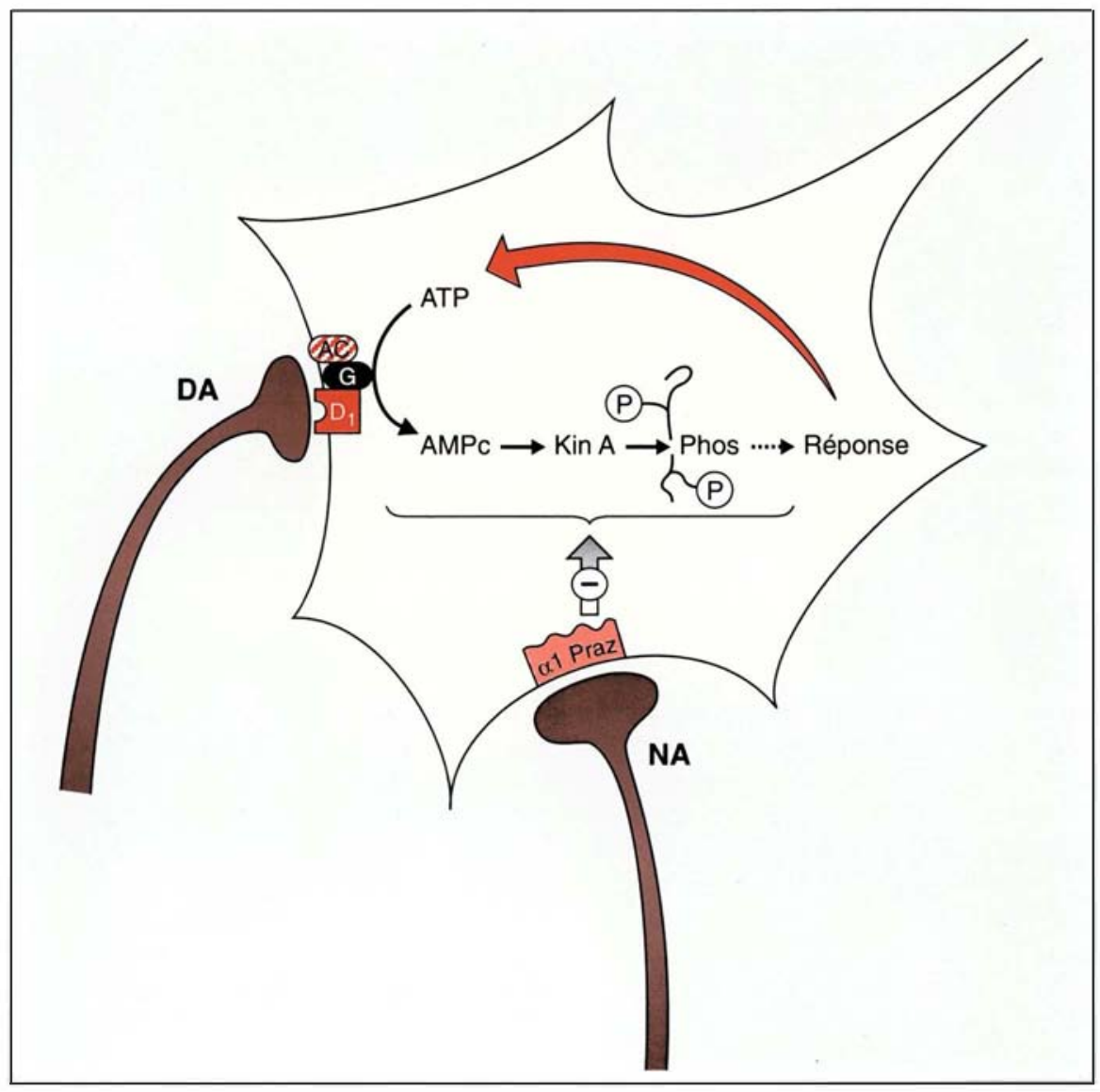

Figure 2. Schéma des interactions entre les récepteurs D1 et $\alpha 1$-adrénergiques dans le cortex préfrontal. Les expériences réalisées sur des cultures embryonnaires de cortex permettent de penser que ces deux récepteurs se trouvent localisés sur les mêmes cellules. Néanmoins, les localisations autoradiographiques de ces sites (respectivement les couches corticales III et VNI pour les sites $\alpha 1$-adrénergiques et $D 1)$ indiquent que ces récepteurs sont situés dans des régions cellulaires différentes (soma, dendrite, axone). La stimulation des récepteurs $\alpha 1$-adrénergiques, en inhibant les réponses induites par la stimulation des récepteurs D1, permettrait le développement d'une hypersensibilité de ces derniers lors d'une dénervation dopaminergique. Le prazosin, en bloquant les récepteurs $\alpha 1$-adrénergiques, faciliterait la transmission dopaminergique corticale de type D1. DA : voie dopaminergique; NA : voie adrénergique ; KinA : protéine kinase $A$, stimulée par l'AMPcy- 
adrénergiques par un agoniste, la méthoxamine, accélère la resensibilisation des récepteurs Dl préalablement désensibilisés par la dopamine [30]. Étant donné l'absence de contacts synaptiques entre les neurones après six jours de culture, cette observation permet d'envisager une colocalisation des récepteurs Dl et $\alpha \mathrm{l}$-adrénergiques sur certains neurones corticaux (figure 2).

L'ensemble de ces expériences suggère que l'hypersensibilité d'un récepteur ne se développe in vivo que lorsqu'est perturbée la cascade d'événements moléculaires que sa stimulation doit entraîner. Dans l'interaction entre les récepteurs Dl et $\alpha \mathrm{l}$ adrénergiques, cela signifierait que la stimulation du récepteur $\alpha$ l-adrénergique inhiberait l'une des étapes enzymatiques découlant de la stimulation des récepteurs $\mathrm{Dl}$ (figure 2). Au contraire, le prazosin, en bloquant l'action de la noradrénaline sur le récepteur $\alpha$ l-adrénergique, faciliterait la transmission dopaminergique corticale relayée par le récepteur DI, évitant ainsi le développement d'une hypersensibilité de ce récepteur.

\section{Conclusion}

Nous venons de décrire deux types d'hétéro-régulation qui ont pu être observés à la suite de l'interruption chronique d'une voie neuronale: (1) Dans le noyau accumbens et la partie antéro-médiane du striatum, les récepteurs D1 sont modulés par les neurones glutamatergiques dont les corps cellulaires se trouvent dans le cortex préfrontal et qui sont euxmêmes sous le contrôle des afférences dopaminergiques corticales. (2) Dans le cortex préfrontal, les récepteurs Dl sont modulés par les fibres noradrénergiques qui stimulent un type de récepteur $\alpha \mathrm{l}$-adrénergique particulièrement sensible au prazosin.

En ce qui concerne l'hétéro-régulation des récepteurs Dl des structures sous-corticales par les fibres glutamatergiques, il est intéressant de se rappeler que des études en microscopie électronique ont mis en évidence des contacts entre les neurones glutamatergiques et dopaminergiques et la population de neurones striataux de taille intermédiaire qui innervent la substance noire [31]. Par ailleurs, des données électrophysiologiques ont indiqué que les neurones dopaminergiques nigro-striataux modulaient les réponses évoquées par la stimulation des efférences corticales [32]. Il est probable qu'une organisation de même type existe dans le noyau accumbens.

En ce qui concerne l'hétéro-régulation des récepteurs $\mathrm{Dl}$ dans le cortex, la récupération de symptômes caractéristiques d'un dysfonctionnement cortico-limbique observée lors d'un blocage des récepteurs $\alpha$ l-adrénergiques corticaux ou de la destruction des fibres noradrénergiques indique l'existence d'un équilibre entre les deux systèmes catécholaminergiques. Cet équilibre semble nécessaire à l'intégrité des fonctions qui découlent des interactions cortico-sous-corticales. Il existe néanmoins une hiérarchie entre les deux systèmes catécholaminergiques puisque le blocage de la transmission noradrénergique, par elle-même, n'induit aucune modification de l'activité locomotrice ou de l'alternance qui semblent modulées par les neurones dopaminergiques. Une hiérarchie identique peut aussi exister dans d'autres structures innervées par les neurones dopaminergiques et noradrénergiques. Il a, en effet, été décrit que des injections de 6-hydroxydopamine dans le septum latéral ne perturbent le comportement d'alternance que si les fibres noradrénergiques sont préservées par la lésion [33]. L'hétéro-régulation des récepteurs Dl permet aussi de retrouver l'antagonisme fonctionnel des transmissions dopaminergiques dans les structures corticales et souscorticales: chez des animaux ayant subi une lésion électrolytique de l'aire tegmentale ventrale, l'hypersensibilité des récepteurs Dl corticaux participe à l'absence d'hypersensibilité des récepteurs Dl sous-corticaux. En d'autres termes, la présence d'un déficit de la transmission dopaminergique corticale libère la transmission dopaminergique sous-corticale et les animaux développent une hyperactivité locomotrice. Au contraire, si les récepteurs Dl corticaux ne sont pas hypersensibles, à la suite d'une interruption de la transmission noradrénergique corticale, les animaux ne sont pas hyperactifs et ne présentent pas de trouble de l'alternance spontanée. Dans ce dernier cas, certains animaux peuvent même présenter une hypersensibilité des récepteurs Dl sous-corticaux et être hypoactifs.

Sur un plan thérapeutique, il est probable qu'une meilleure connaissance des récepteurs glutamatergiques sous-corticaux qui interviennent dans la modification de la transmission dopaminergique sous-corticale permettra d'améliorer les déficits moteurs liés à la dégénérescence des fibres dopaminergiques nigro-striatales.

Par ailleurs, compte tenu de l'importance fonctionnelle des neurones dopaminergiques méso-corticaux dans le contrôle de l'émotivité, un traitement systémique susceptible d'atteindre spécifiquement la transmission dopaminergique corticale paraît particulièrement intéressant. L'interaction que nous avons mise en évidence avec les récepteurs $\alpha$ l-adrénergiques corticaux représente sans doute une possibilité de modifier de façon préférentielle la transmission dopaminergique corticale. En effet, les études sur les cultures cellulaires ont montré que cette interaction noradrénaline/dopamine n'existait pas sur les neurones striataux [30]. En outre, l'analyse autoradiographique de la distribution des récepteurs $\alpha$ l-adrénergiques sensibles au prazosin indique que ces sites, présents dans la couche III du cortex, n'apparaissent pas dans le striatum et le noyau accumbens [27]. Enfin, il est intéressant de noter que certains effets complémentaires des produits psychotropes comme les neuroleptiques ou les antidépresseurs (phénothiazines, imipraminiques) semblent en relation avec leurs propriétés antagonistes vis-à-vis des récepteurs $\alpha$ l-adrénergiques. Ces effets pourraient être précisément dus à leur action sur la transmission dopaminergique corticale

\section{TIRÉS À PART}

J.P. Tassin. 


\section{Summary \\ Receptor heteroregulation : an indication of functional relation- ships between neuronal systems}

To study the functional interrelations between the ascending dopaminergic (DA) systems, we have focused our attention on the regulation of one type of DA receptor, the Dl receptor, which is positively coupled to adenylate cyclase. The initial experiments revealed that the sensitivity of D1 receptors was not only regulated by afferent DA fibres but also by neurotransmitters released by non-DA nerve terminals. Cortical as well as subcortical Dl receptors seem to be subject to this heteroregulation process. Analyses of the nature and the origin of the non-DA fibres implicated in the heteroregulation of D1 receptors indicated that these neurons were part of well-defined anatomical circuits and suggested that this heteroregulation had functional significance. An intact cortical DA innervation appeared to be of major importance to obtain a denervation hypersensitivity of the Dl receptors in the nucleus accumbens as well as in the striatum. This indicated that the DA stimulation of cortical or subcortical structures have opposite effects on functions regulated by these structures. Behavioural experiments have indeed confirmed the antagonistic functional roles of cortical and subcortical DA innervations. In the prefrontal cortex, sensitivity of D1 receptors appears to be regulated by ascending noradrenergic (NA) fibres. The physiological role of these ascending NA fibres, and more particularly of the $\alpha 1$-adrenergic receptors, has been confirmed in behavioural experiments which indicated that the deficits induced by the destruction of the DA mesocortical pathway could be compensated by the superimposed destruction of the ascending NA fibres or by the pharmacological blockade of the $\alpha 1$-adrenergic receptors by prazosin. 